\title{
Detection of mucin-hypersecreting bile duct tumor by direct peroral cholangioscopy
}

Detection of the rare mucin-producing bile duct tumor is difficult because of ambiguity caused by the abundant mucin secretion and/or by the superficial mucosal spread of the tumor along the bile duct [1]. We report the case of a 77-year-old patient, who initially presented with severe jaundice, right upper abdominal pain, and fever. The endoscopic retrograde cholangiopancreatography (ERCP) demonstrated a large amount of viscous mucin in a dilated bile duct system ( Fig. 1 a,b).
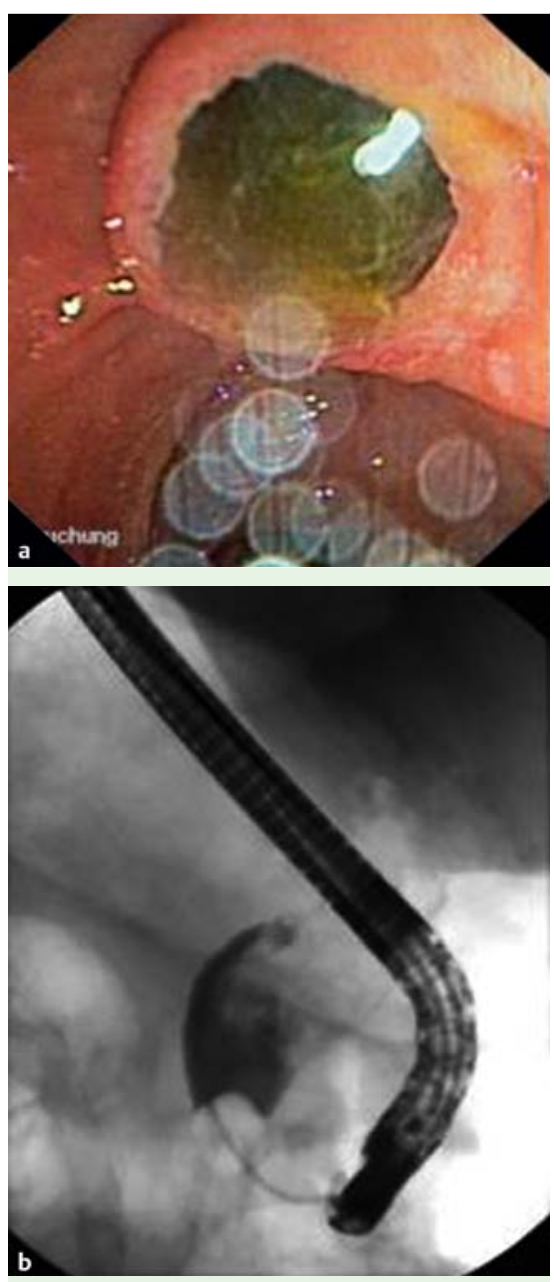

Fig. 1 a Viscous mucin in the ampulla of Vater visualized during endoscopic retrograde cholangiopancreatography (ERCP). b Radiograph taken during endoscopic retrograde cholangiopancreatography (ERCP) showing an inhomogeneous distribution of contrast medium in the dilated bile duct.
A large endoscopic sphincterotomy was initially performed but the mucin could not be completely removed with basket and balloon catheters and a tumor could not be detected. Cholangioscopy by babymother technique was also unhelpful; suction power and pressure washing were insufficient and devices such as basket catheters are not available for the choledochofiberscope (CHF-BP30, 1.2-mm working channel; Olympus, Tokyo, Japan).

It was decided to use an ultrathin upper endoscope (GIF-XP160, outer diameter of $5.9 \mathrm{~mm}, 2.0-\mathrm{mm}$ working channel; Olympus, Tokyo, Japan) to perform direct peroral cholangioscopy ( Fig. 2).

This procedure, first reported in 1977, has since been described with several variations [2-5]. After insertion of a nasal biliary drainage tube (PBD-21Z, 2.35-mm maximum diameter; Olympus, Tokyo, Japan) the ultrathin upper endoscope was advanced beside the drainage tube directly into the bile duct by pushing and pulling in a stepwise manner while twisting the endoscope. Using an endowasher (Aqua:Master; Endo-Technik W. Griesat $\mathrm{GmbH}$, Solingen, Germany) via the working channel of the ultrathin endoscope, it

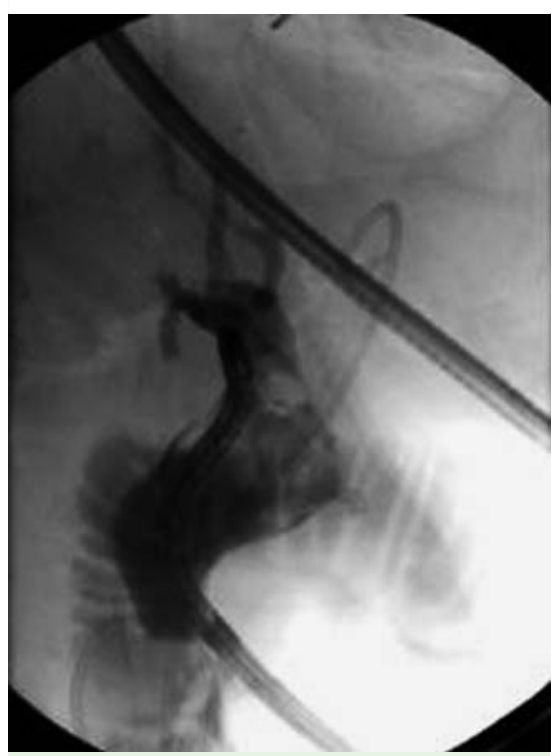

Fig. 2 Radiograph taken during direct peroral cholangioscopy showing an ultrathin upper endoscope positioned in the bile duct of the left hepatic lobe and nasal biliary drainage tube in the right hepatic lobe. was possible to wash out the mucin from the bile duct and to have a direct view of the tumor ( Fig. 3 ).

Biopsies confirmed the diagnosis of a mucin-producing bile duct adenoma in the left hepatic lobe. The patient underwent
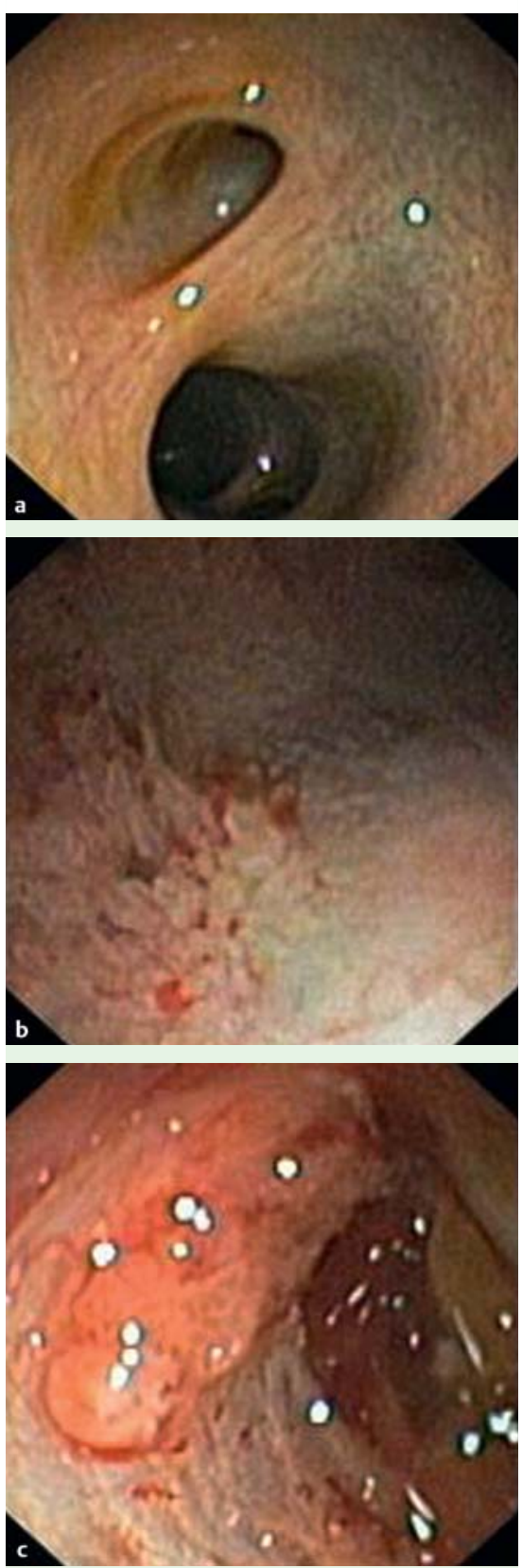

Fig. 3 Endoscopic views using the ultrathin endoscope: a normal bile duct in the right hepatic lobe; $\mathbf{b}$ tubular neoplasm in the bile duct of the left hepatic lobe; $\mathbf{c}$ tumor protruding into the bile duct of the left hepatic lobe. 
successful resection of the tumor. In this case direct peroral cholangioscopy was the only endoscopic technique able to visualize the mucin-producing tumor.

\section{Endoscopy_UCTN_Code_CCL_1AZ_2AC}

Competing interests: None

I. Wallstabe ${ }^{1}$, I. Schiefke ${ }^{1}$, P. Lamesch ${ }^{2}$

1 Department of Gastroenterology and Hepatology, Klinikum St. Georg, Leipzig, Germany

2 Department of General and Visceral Surgery, Klinikum St. Georg, Leipzig, Germany

\section{References}

$1 \mathrm{Kim}$ HJ, Kim MH, Lee SK et al. Mucin-hypersecreting bile duct tumor characterized by a striking homology with an intraductal papillary mucinous tumor (IPMT) of the pancreas. Endoscopy 2000; 32: 389-393

2 Urakami Y, Seifert E, Butke H. Peroral direct cholangioscopy (PDCS) using routine straight-view endoscope: first report. Endoscopy 1977; 9: 27 - 30

3 Choi HJ, Moon JH, Ko BM et al. Overtube-balloon-assisted direct peroral cholangioscopy by using an ultra-slim upper endoscope. Gastrointest Endosc 2009; 69: 935-940

4 Mori A, Tatebe H, Ohashi T et al. Balloon-assisted insertion of a cholangioscope into the common bile duct: a novel technique. Endoscopy 2008; 40: E106-E107

5 Tsou YK, Lin CH, Tang JH et al. Direct peroral cholangioscopy using ultraslim endoscope and overtube balloon-assisted technique: a case series. Endoscopy 2010; 42: 681-683

\section{Bibliography}

DOI 10.1055/s-0030-1256298

Endoscopy 2011; 43: E180 -E181

(c) Georg Thieme Verlag KG Stuttgart · New York . ISSN 0013-726X

\section{Corresponding author \\ I. Wallstabe, MD}

Department of Gastroenterology and Hepatology Klinikum St. Georg

Delitzscher Str. 141

04129 Leipzig

Germany

Fax: +49-341-9092673

wallstabe@endoskopieren.de 